\title{
Region-specific differences in colorectal cancer: Slovakia and Hungary have highest incidence in Europe
}

\author{
Simko $V^{1}$, Ginter $E^{2}$ \\ State University of New York, Downstate Medical Center, Brooklyn, USA. simko 2@verizon.net
}

\begin{abstract}
Epidemiological data on colorectal cancer (CRC) exhibit high incidence in Central East Europe. Hungary, Slovakia and Croatia represent the lead. For decades it was the Czech Republic but it attained the fourth rank after the mid-2000. Remarkably, the Ashkenazi Jews who imigrated to the USA from Central Europe have the highest incidence of CRC among US minorities. They also have high incidence of inflammatory bowel disease, a risk for CRC. Notably, countries surrounding the Central European focus of CRC, Austria, Germany, Poland, Romania, Ukraine and Russia have substantially lower incidence. CRC in Central Europe has higher incidence than CRC among the highest at-risk cohort in the USA, the elderly blacks. Research and the genome wide screening identified genetic mutations associated with CRC in Ashkenazis from Central Europe. Some risk factors for CRC are non genotypic as evidenced by wide variation in CRC incidence in the course of only a few decades. Recent trends offer hope that identification of the non-innate pathogenic mechanisms would potentially reduce the burden of this third most lethal malignancy (Tab. 1, Fig. 4, Ref. 40). Text in PDF www.elis.sk. KEY WORDS: colorectal cancer, inflammatory bowel, Hungary, Slovakia, Croatia, Ashkenazi Jews of Central Europe, genotypic and innate risk, probiotic immunomodulation of intestinal cells.
\end{abstract}

\section{Introduction}

CRC is the third most common cancer in US men, after lung and prostate. It is the second most common malignancy in women, after breast cancer (1, 2). CRC varies based on patient demographics. The aim of this report is to highlight remarkable differences in European cancer incidence with special attention to Hungary, Slovakia and Croatia (Fig. 1). It is revealing to project data of CRC in Central Europe on CRC in other European populations, on the US whites, blacks and on Ashkenazi Jews in the USA who originate from Central Europe (3). Remarkably, Ashkenazi from Ukraine and Belarus do not exhibit such high CRC. The populations of Austria, Poland, Romania, Ukraine and Russia also do not share such high burden of CRC. This removes former Austro- Hungary as a historic target to blame for CRC.

High incidence of CRC in Hungary, Slovakia and Croatia is region- specific and unrelated to the devastating health mismanagement of past communism $(4,5)$. Croatia with a high incidence of CRC experienced a "softer", non-soviet brand of communism. Cardiovascular disease, alcoholism, suicides and low life expectancy are typical for former Soviet Union, yet the incidence of CRC in Russia is only about $60 \%$ compared with Hungary (Tab. 1). Neither smoking appears as an important risk for CRC in Central Europe. Balkan countries have high addiction to smoking. Bul-

${ }^{1}$ State University of New York, Downstate Medical Center, Brooklyn, USA, ${ }^{2}$ Slovak Medical University, emeritus, Bratislava, Slovakia

Address for correspondence: V. Simko, MD, PhD, AGAF, FACG, 102 Signal Hill Rd, Staten Island, NY 10301, USA.

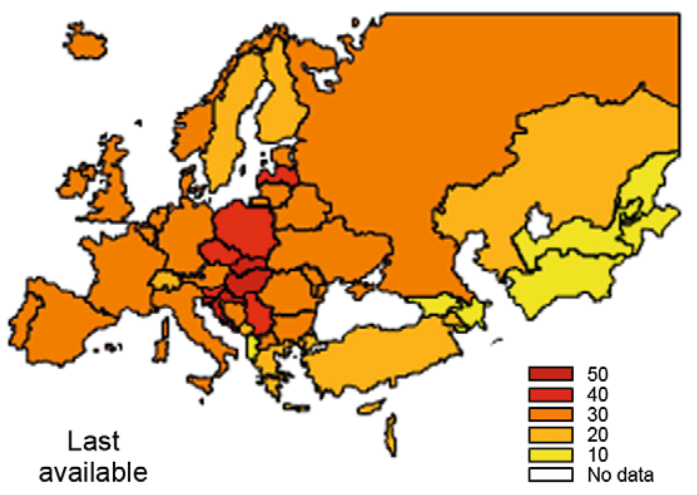

Fig. 1. Highest incidence of colorectal cancer in Europe predominates in Central East Europe, in Hungary, Slovakia, Croatia and in the Czech Republic (Ref. 2: Europe Health for All Database (HFA-DB) Copenhagen, WHO Regional Office for Europe, 2014).

garia has up to $47 \%$ of regular smokers, while Croatia with very high incidence of CRC has $34 \%$.

Risk of CRC progressively increases with age. Data from the Surveillance Epidemiology and End Results (SEER) registry in the USA revealed age specific incidence of CRC. Yet, this malignancy in both white and black Americans at age 55-59 is substantially lower than in the Central East Europe. This is true even for blacks aged over 80 years. This highest risk cohort of blacks has CRC incidence 30.8 per 100,000 (6), in comparison, Hungary has incidence 49.3, Slovakia 43.5. The CRC rates in Czech women 
Tab. 1. Trends in colorectal male cancer mortality in the past two decades manifest highest incidence in Hungary, Slovakia and Croatia with a remarkable decline in the Czech Republic. Less affected are Austria, Poland, Russia and Ukraine (Ref. 2).

\begin{tabular}{|c|c|c|c|c|c|c|c|c|c|c|c|}
\hline Year & Austria & Croatia & Czech R. & Germany & Hungary & Israel & Poland & Romania & Russia & Slovakia & Ukraine \\
\hline 1986 & 34.0 & 25.6 & 51.3 & - & 40.9 & 21.5 & 19.7 & 13.8 & 23.7 & - & 20.9 \\
\hline 1992 & 36.9 & 28.5 & 53.3 & 34.5 & 49.0 & 24.0 & 22.8 & 14.7 & 26.2 & 36.5 & 25.6 \\
\hline 2000 & 30.0 & 46.2 & 52.1 & 30.2 & 50.8 & 25.6 & 28.2 & 18.9 & 28.5 & 47.5 & 26.9 \\
\hline 2005 & 26.5 & 41.3 & 46.8 & 26.1 & 47.6 & 24.3 & 29.1 & 23.2 & 29.8 & 47.1 & 27.7 \\
\hline 2010 & 21.0 & 43.0 & 38.7 & 22.3 & 49.3 & 20.1 & 31.1 & 26.3 & 29.6 & 43.5 & 27.6 \\
\hline
\end{tabular}

SDR, males, Malignant neoplasm of colon, rectum and anus, per 100000.

and in Japan have exceeded the incidence reported in women in the USA, Canada and Australia (7).

Ethnic differences have also been noted in expression of other malignancies: prostate, breast, gastric and esophageal cancer (8). Recognition of ethnic and racial disease predisposition is not racism but an ethical diagnostic tool, assisting in revealing disease pathogenesis and in proposing effective disease management.

Biological challenge of high incidence of CRC in Central East Europe has to be deciphered using genetic and epigenetic research. This review analyzes potential genetic and epigenetic risks for CRC, including inflammatory bowel disease (IBD) and the role of diet. Nutrients immunomodulate the intestinal microbiota, potentially contributing to malignant intestinal cell transformation.

There is a reason for tempered optimism. Changing trends in CRC incidence in recent decades suggest the presence of important non genetic environmental mechanisms in the pathogenesis of CRC. Public awareness of health burden of CRC is essential. The potential for reducing consequences of CRC malignancy may thus be substantial (9). Preventive measures and early detection lead to a much better prognosis in patients diagnosed early: progression from detectable precancerous lesions to advanced CRC is slow.

\section{Regional and ethnic epidemiology of colorectal cancer}

Extensive survey of epidemiology in malignant disorders in Central and South Eastern Europe covering the past 20-40 years (Figs 2, 3, 4) confirmed wide regional differences (10). CRC was noted to be prominent in Hungary, Slovakia, Czech Republic and Croatia. High incidence of CRC results in specific mortality in these countries to be twice or three times higher than in nearby countries with low incidence, Bosnia, Greece and Albania. Time trends in CRC in the Czech Republic and neighboring countries demonstrate diverse patterns in the last two decades (11).

Each year approximately 8,000 people are diagnosed in the Czech Republic with CRC and about 4,000 die from this malignancy (12). Czech CRC screening registry was established in 2006. Screening program including fecal occult blood testing and preventive colonoscopy covers about $25 \%$ of the target population. These measures might have been associated with the decline in Czech CRC incidence observed in the past decade. Czech CRC declined by $25 \%$ while it increased in Poland by $21 \%$ and in Romania by $57 \%$. Statistics undoubtedly depend s on the quality of diagnosis and reporting.

Some reports on CRC cover only selected regions, mostly in Western Europe. Eurocare represents research collaboration between several European population - based registries that started

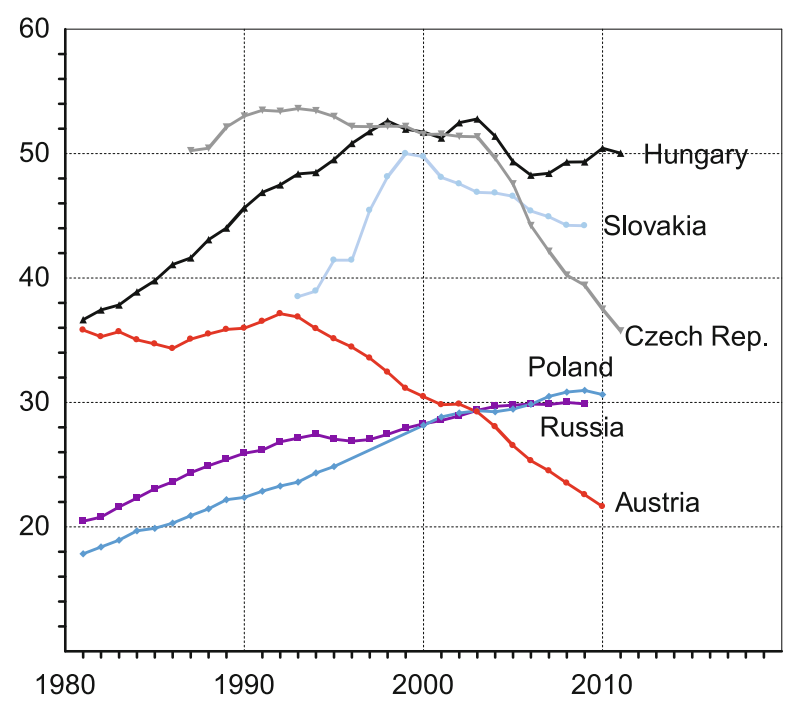

Fig. 2. Trends in male colorectal cancer mortality in Central East Europe in 1980-2010 (Ref. 2).

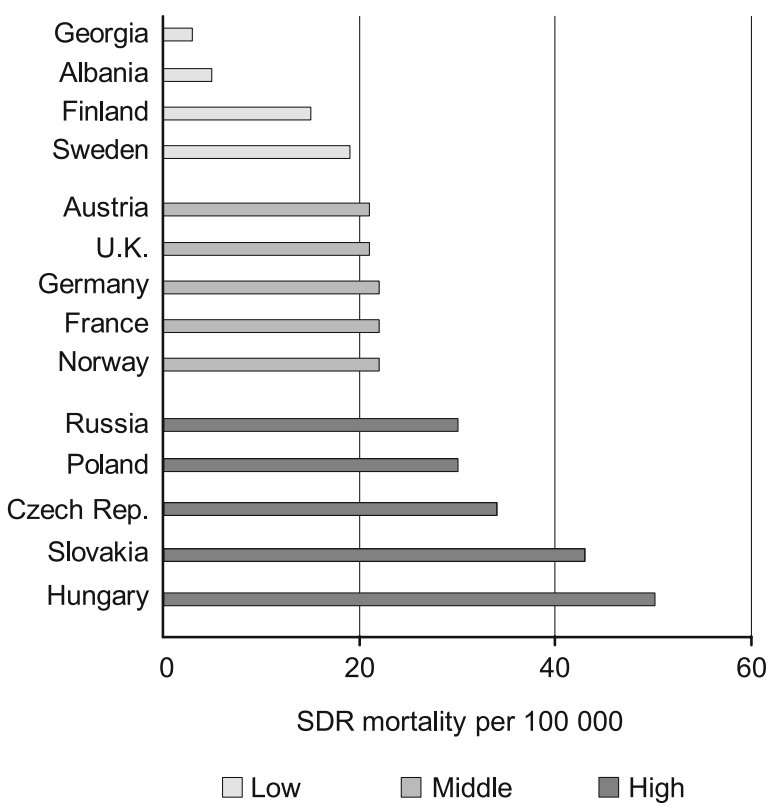

Fig. 3. Recent male mortality for colorectal cancer in 13 European countries (Ref. 2).

in 1990. There has been considerable variation in age-adjusted five year survival by country and region (13). Highest CRC survival rates were in Northern Europe, lowest in Eastern Europe. The first 


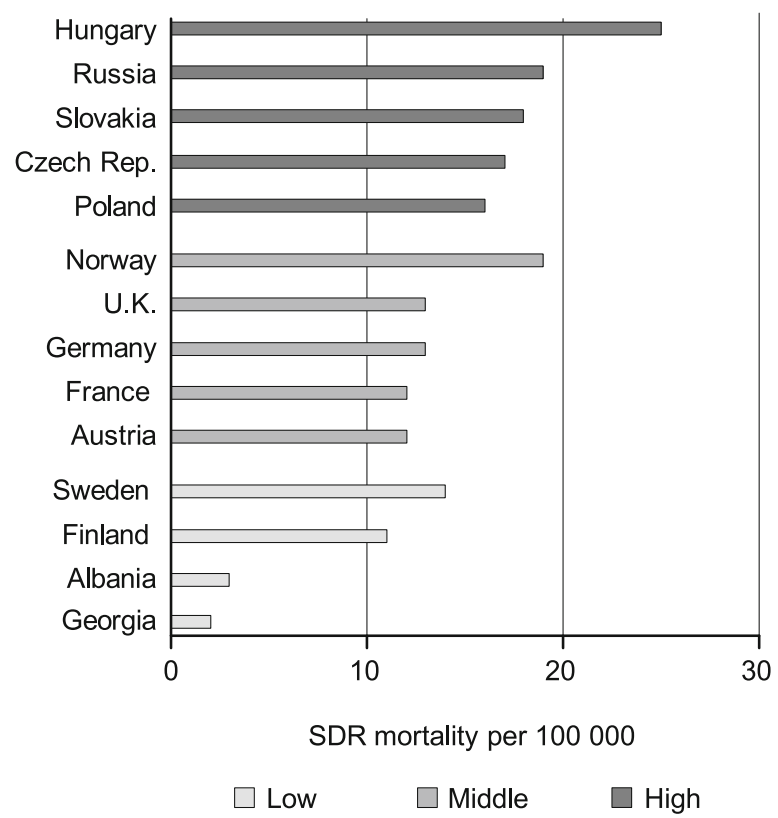

Fig. 4. Recent female mortality for colorectal cancer in 13 European countries (Ref. 2).

European country to implement an organized CRC screening program was Germany in 1976.

Another potentially predictable tool in CRC epidemiology is based on CRC clinical presentation. Attempts to compare different geographic regions on the basis of CRC staging are incomplete and do not include all the high risk countries in Europe (14). Equally difficult is interpretation of difference in CRC based on surgery leading to cancer resection and patient survival. There may be difference in tumor location related to the ethnic group. Even at an advanced metastatic stage, patients with CRC distal to the splenic flexure have twice better prognosis than the right colonic tumors. US patients with CRC have more frequently surgical resection compared to other countries. The three year survival appears to be higher for the US than for most European patients. This may also be related to CRC staging at the first diagnosis.

Each year 140,000 people in the US are diagnosed with CRC (15). Most of these will be classified as sporadic, but estimates suggest that $5-6 \%$ of CRC develops as a result of inherited gene mutation. Critically important is early diagnosis and the cancer stage: of the four stages, 1-2 stage is diagnosed in about $39 \%$ of US patients, when cancer is still localized and carries up to $85 \%$ survival at five years (16).

Marked ethnic differences in CRC have been reported comparing Caucasian (white) population with African Americans (blacks) and Asians in the US. CRC incidence and mortality is higher in blacks than in whites (6).

Differences were also observed in the prevalence and location of large colon polyps and tumor location based on age, sex and ethnicity $(17,18)$. Racial disparity in colon cancer may be due, in part, to biological differences in tumor type (19). Prevalence of microsatellite instability in CRC histological samples was $7 \%$ among American blacks compared with $14 \%$ among white patients.

In contrast to innate genetic mechanisms in CRC, acquired and environmental factors are suggested by the recent variation in the incidence of CRC in the United States where this malignancy declined in both men and women since 1975, particularly in people over the age of 75 (20). This retrospective cohort of over 393,000 patients from 1975 to 2010 also revealed a $2 \%$ increase among those aged $20-34$ years.

CRC in Ashkenazi Jews is intriguing when comparing their high CRC incidence with CRC in Central East Europe. Ashkenazi Jews resided mostly in Germany and Austro-Hungary. Relatively later they extended their diaspora to Poland, Ukraine and Belarus. They differ in ethnicity from Sephardic Jews who originated predominantly at the Iberian Peninsula. It is the Ashkenazi Jews with origin from Central Europe who have the highest incidence of IBD and CRC among all Jews (21). Curiously, CRC is less frequent in Ashkenazis from Poland and Ukraine. This observation has been confirmed in Jews living in the United States.

This suggests the presence of important genotypic factors contributing to high CRC in Central East Europeans. These may have been preserved in Ashkenazi from Central Europe even for several generations, after they moved out of the European location. Tracking CRC in Jewish patients may prove revealing in analyzing results of genome wide screening that compared Jewish communities from around the world (22).

Jewish communities in Europe and in the Middle East share many genes inherited from ancestral Jewish population that lived in the Middle East some 3,000 years ago. The Ashkenazis first appeared in Northern Europe around 800 A.D. They thrived in Northern and Eastern Europe until their devastation by Hitler. Now they live mostly in the US. Their shared genetic elements suggest that members of any Jewish community are related to one another about ten times more closely than to any non Jewish people chosen at random. Women of Ashkenazi descent in the United States proved in a random genetic testing positive for cancer- causing genetic mutations (20). Ashkenazis from Central Europe may present a more preserved sample of CRC genotype than the non-Jewish population in these countries.

\section{Inflammatory bowel disease and colorectal cancer}

Chronic intestinal inflammation is well established as a risk factor for CRC. The mechanism for this association remains unclear (23). IBD is comprised of two major phenotypes, ulcerative colitis and Crohn's disease. Regional and ethnic differences in IBD may offer the answer to high incidence of CRC in Central East Europe.

There have been numerous experimental studies suggesting that inflammatory cells and their associated mediators form a microenvironment favoring the development of CRC, most likely by enhancing DNA damage in epithelial cells (24). Susceptibility to IBD is partly determined by genes encoding immune responses which are triggered by environmental stimuli.

Intestinal microbial environment has the potential to modulate the development of CRC. Intestinal microbiota may promote 
intestinal tumorigenesis by the expansion of microorganisms with genotoxic capabilities (23). Intestinal barrier dysfunction may open bacterial access to the mucosa leading to increased immunoreactivity, promoting contact of host cells with carcinogenic products.

The type of microorganisms encoding the genotoxic peptide may vary, being present in some individuals and not in others. Genetic alterations may be associated with shifts in microbiota population. Without doubt, the role of diet and of natural prebiotics and probiotics enters this pathogenetic equation. Some food products were confirmed to reduce or enhance E. coli attachment to the intestinal mucosa (24). Dietary habits affect gut microbial population and predisposition to malignancy.

Epidemiological literature based on twenty eight publications on geographic distribution in IBD revealed marked phenotypic and ethnic differences (25). Compared with African Americans, greater proportion of Hispanics and Asians were diagnosed with ulcerative colitis than with Crohn's (26). Marked phenotypic racial differences were revealed also in anatomic location of IBD expression. African Americans compared with other US ethnic groups had higher predisposition for Crohn's in the colorectum and at the perianal area but less frequently in the terminal small bowel (27).

Central European region as a geographic host of predisposition for colorectal disease is prominent also in epidemiology of IBD. Ulcerative colitis and Crohn's are more common among the Jews than in non - Jews. The incidence and prevalence of IBD is two-to-four fold higher in Ashkenazi Jews when compared to non- Jewish Caucasians. IBD accounts to $1-3 \%$ of all cases of CRC in the Ashkenazis (28). The molecular pathway leading to CRC in IBD appears to differ from the more prevalent adenoma - (polyp) sequence resulting in malignancy.

Epidemiological analysis of 233 US Jewish patients with IBD found significantly more Jews of Central European origin relative to individuals of Polish and Russian descent (3). This suggests that IBD genes are more prevalent in the Jewish population that originated in middle Europe than in Poland and Russia. It may indicate a genetically predisposed geographic subset of Jewish population at risk.

NOD2/CARD15 has been identified as a major susceptibility gene for Crohn's disease. Significance of the NOD2/CARD15 mutations carrying an increased risk of IBD was confirmed in Jewish Crohn's disease patients in Israel (29). Higher frequency of CARD15 mutations was found in Ashkenazi patients with Crohn's disease from Central (44 \%) versus Eastern (24 \%) Europe (30). In general, Ashkenazis appeared to have higher frequency of CARD15 mutations than the Sephardic Oriental Jewish controls. These genetic mutations also contribute to higher incidence of IBD among Ashkenazi Jews.

A report from Hungary (31) interestingly connects with the genetic studies on IBD of other authors. Studies in Eastern European individuals, a large cohort of 652 Hungarian patients with IBD, indicate a serological genotype marker NOD2/ CARD15 to be associated with more complicated disease phenotype, potentially requiring surgical intervention.
Research on association between TNF-SF 15 haplotype and severity of IBD observed higher frequency of the protective haplotype in non Jewish, compared with the Jewish patients (32).

Better understanding of IBD epidemiology and its relation to CRC may come from intensive genome- wide research to link specific genetic loci with phenotypic traits in individuals at risk.

\section{Potential pathogenetic factors in regional differences of colorectal malignancy}

CRC is a biologically heterogenous disease that can develop via a number of distinct pathways, involving different combinations of genetic and epigenetic changes (33). Variability in etiopathogenetic alterations can translate to differences in predisposition and survival in CRC (34). Genetic differences in tumor biology may be responsible for racial disparity in CRC. The prevalence of microsatellite that generally predicts better outcome was only $7.4 \%$ in African Americans (higher incidence of CRC) compared to $14 \%$ among the white US patients (19).

Past three decades marked an important progress in the identification of somatic genes and epigenetic factors present in CRC (35). Still, the extensive complexity and diversity in CRC genomes and epigenomes contribute to difficulty in clarification of the ethnic factors in CRC.

The accumulation of multiple loss-of-function defects in tumor suppressor genes, and gain-of-function defects in selected tumor suppressor genes combines with epigenetic mechanisms, including changes in DNA methylation. Strategies attempting to define molecular alterations in CRC may prove useful in revealing cancer initiation, response to conventional and novel therapy, cancer recurrence and overall outcome (35).

Regarding acquired CRC expression, multiple factors have to be considered. It is not only the modifying effect of the diet, dietary antioxidants, vitamin D stores, the intestinal microbiota but also socioeconomic mechanisms. These include availability of CRC screening and diagnosis, quality of health care, as well as public awareness of disease symptoms.

Colorectal epithelium is the site of origin of CRC. The integrity of colonic mucosa depends on coordination of the epithelium, luminal microorganisms and the epithelial immune system. This is oriented for innate and adoptive recognition of microbial antigens.

Intestinal epithelial cells normally provide an efficient barrier to exclude adverse enteric bacteria possessing their immunologically active adjuvants, from interacting with immune cells in the lamina propria (36). In health, host commensal bacteria activate a response by epithelial plasma cells, macrophages, T lymphocytes and beta cells, to permit coexistence with potentially toxic microbial products. Induction of protective molecules stimulates defensive mechanisms that enhance the function of the mucosal barrier. Protective capacity of the mucosa is balanced against the tissue damage produced by microbial immune activity, with the ultimate aim to preserve normal intestinal function (37).

Research on dynamic interaction of commensal microorganisms with the host environment (36) has the potential to reveal geographic and ethnic differences in CRC. Intake and availability 
of protective nutrients may vary among populations that manifest different predisposition to CRC. Essential role of population diet and of potential manipulations affecting the gut microbiome (antibiotics, prebiotics and probiotics) (38) appears a challenging subject in studying geographic predisposition to CRC. Documented environmental factors shaping the gut microbial environment include peripartum transfer of maternal microbiota and the benefit of breast feeding, instead of using sterile commercial dietary supplements. Almost $90 \%$ of the colorectal tumors originates sporadically, genetic predisposition or familiar oncological burden takes place in only $5 \%$. The most important exogenic factor is dietetary regime of the individual. High volume of vegetable diet has protective effect, because of the containment of anti-tumorous substances (carotenoids, folates, phenols, flavonoids). Consumation of fructo-oligosacharides selectively improves growth and activity of potentially beneficial bacteria (lactobacilli, bifidobacteria). The risk of tumor formation is decreased with non-digestable fiber and carotenoids. Regular body activity and physical training is also considered as a protective factor. One of the highest risk factors are consumation of red meat, which increases the stimulation of biliary acids and developement of carcinogenous nitrosamine. Obesity also doubles this risk. In developement of colorectal cancer also known is the synergic effect of smoking and alcohol abuse.

Several specific nutritional factors have been proposed to affect gut integrity. Higher tissue stores of vitamin D were reported to be associated with reduced risk of CRC (39). Vitamin D may interact with the immune system, boosting its protective response to tumor growth. However, recent reviews have cast doubt on any causal link between vitamin D deficiency and non skeletal health. Vitamin D deficiency may just be a marker of ill health.

Diet poor in folic acid and in vitamin B6, reduced intake of calcium and of milk products have also been associated with higher risk of CRC (40). This extensive list also was suggested to include statins and aspirin medication. And, also in the inventory of potential culprits, there is the usual reference to factors of life style, adverse effect of physical inactivity, overweight, smoking, alcohol and processed food .

It is hard to believe that individuals in Hungary, Slovakia, Croatia and Ashkenazi Jews living in the United States as a second or third generation, would share simple unifying causative factors, related to a dietary component or to difference in life style. Serious research, molecular biology and genetic studies are the potential key to unravel the enigmatic predisposition of Central East Europeans for CRC. Exploration of CRC pathogenesis in Central East Europe may yield key insights into genetic and environmental risk factors which may be applicable to all ethnicities.

In conclusion, high incidence of colorectal cancer in CentralEast Europe, in Hungary, Slovakia and Croatia represents a remarkable scientific challenge. Crohn's disease, risk factor in CRC, varies greatly in terms of geographic incidence, clinical severity and pathological findings. Genotypic predisposition to CRC and to inflammatory bowel associated with CRC in American Ashkenazi Jews who originated from Central East Europe, is further adding weight to this interesting dilemma. It suggests the existence of an important genotypic component present and acting in this speci- fic geographic distribution of CRC. However, temporal variation in CRC, an impressive decline of this malignancy in the Czech population, indicates relevant environmental influences, capable to modify the CRC phenotype. There is a hope that when both the genotypic and the non- innate causes of CRC variability are identified, medicine will be able to implement preventive measures effective against this feared malignant scourge.

\section{References}

1. Ferlay J, Shiu HR, Brag F et al. Estimates of worldwide burden of cancer in 2008: Globocan 2008. Int J Cancer 2010;127: 2893-2917.

2. Health for All. Database (HFA-DB), Copenhagen, WHO Regional Office for Europe, 2014.

3. Roth MP, Petersen GM, McElree C et al. Geographic origins of Jewish patients with inflammatory bowel disease. Gastroenterology 1990; 99: 286-287.

4. Ginter E, Simko V. Health of Europeans twenty years after the fall of Berlin Wall. Bratisl Lek Listy 2010; 111: 398-403.

5. Ginter E, Simko V. Negative impact of communism on Eastern Europe population health. Medicina Interna 2010; 7: 55-60.

6. Laiyemo AO, Doubeni CA, Pinsky PF et al. Race and colorectal cancer disparities: Health care utilization versus different cancer susceptibilities. J Nat Cancer Inst 2010; 102: 538-546.

7. Tarraga Lopez PJ, Albero JS, Rodriguez Montes JA et al. Primary and secondary prevention of colorectal cancer. Clin Med Insights Gastroenterol 2014; 7: 33-46.

8. Wu X, Chen VW, Andrews PA et al. Incidence of esophageal and gastric cancer among Hispanics, non-Hispanic whites and non-Hispanic blacks in the United States: subsite and histologic differences. Cancer Causes Control 2007; 18: 585-593.

9. Sha Tao, Hoffmeister M, Brenner H. Development and validation of a scoring system to identify individuals at high risk for advanced colorectal neoplasms who should undergo colonoscopy screening. Clin Gastroenterol Hepatol 2014; 12: 478-485.

10. Vrdoljak E, Wojtukiewicz M, Pienkowski T et al. Cancer epidemiology in Central and South Eastern European countries. Croat Med J 2011; 52: 478-487.

11. Zavoral M, Suchanek S, Zavada F et al. Colorectal cancer screening in Europe. World J Gastroenterol 2009; 15: 5907-5915.

12. Zavoral M, Suchanek S, Majek O et al. Colorectal cancer screening: 20 years of development and recent progress. World J Gastroenterol 2014; 20: 3825-3834.

13. Brenner H, Bouvier AM, Froschi R et al. Eurocare Work Group Progress in colorectal cancer survival in Europe from the late 1980 to the early 21st century: the Eurocare study. Int J Cancer 2012; 131: 1649-1658.

14. Cicolallo L, Capocaccia R, Coleman MP et al. Survival differences between European and American patients with colorectal cancer: role of stage at diagnosis and surgery. Gut 2005; 54: 268-273.

15. Stoffel EM, Chitenden A. Genetic testing for hereditary colorectal cancer: Challenges in identifying, counseling and managing high- risk patients. Gastroenterol Hepatol News 2007; 1: 29-39.

16. Henrikson NB, Webber EM, Goddard KA et al. Family history and the natural history of colorectal cancer: systemic review. Genet Med 2015; Jan 15: doi 10.1038. 
17. Shavers VL. Racial/ethnic variation in the anatomic subsite location of in situ and invasive cancer of the colon. J Natl Med Assoc 2007; 99: 733-748.

18. Lieberman DA, Lucas Williams J, Holub JL et al. Race, ethnicity, and sex affect risk for polyps $>9 \mathrm{~mm}$ in average risk individuals. Gastroenterology 2014; 147: 351-358.

19. Fidelia-Lambert M, Grady WM, Carruthers JM. Genomic and epigenetic instability in colorectal cancer pathogenesis. Gastroenterology 2008; 135: 10791099.

20. Bailey CE, Hu CY, You YN et al. Increasing disparities in the age- related incidence of colon and rectal cancers in the United States, 1975-2010. JAMA Surg 2015; 150: 17-22.

21. Atzmon G, Ostrer H, Hao L et al. Abraham's children in the genome era: major Jewish diaspora populations comprise distinct genetic clusters with shared Middle Eastern ancestry. Am J of Human Genetics 2010; 86: 850-859.

22. Levy-Lahad E, Lahad A, Eisenberg $\mathrm{S}$ et al. A single nucleotide polymorphism in the RAD 51 gene modifies cancer risk in BRCA2 but not BRCA1 carriers. Proc Ntl Acad Sci 2001; 98: 3232-3236.

23. Arthur JC, Perez-Chanona E, Muehlbauer $M$ et al. Intestinal inflammation targets cancer - inducing activity of the microbiota. Science 2012; 338: 120-123.

24. Vetrano S, Danese S. Colitis, microbiota, and colon cancer: An infernal triangle. Gastroenterology 2013; 144: 461-463.

25. Hou JK, El-Serag H, Thirumurthi S. Distribution and manifestation of inflammatory bowel disease in Asians, Hispanics, and African Americans: a systematic review. Am J Gastroenterol 2009; 104: 2100-2109.

26. Dama OM, Jahann DA, Reznik R et al. Phenotypic manifestations of inflammatory bowel disease differ between Hispanics and non-Hispanic whites: Results of a large cohort study. Am J Gastroenterol 2013; 108: 231-239.

27. Nguyen GC, Torres EA, Regneiro $M$ et al. Inflammatory bowel disease characteristics among African Americans, Hispanics, and nonHispanic Whites: characterization of a large North American cohort. Am J Gastroenterol 2006; 101: 1012-1023.

28. Lynch HT, Brand RE, Locker GY. Inflammatory bowel disease in Ashkenazi Jews: implications for familial colorectal cancer. Fam Cancer 2004; 3: 229-232.
29. Karban A, Waterman M, Panhuysen C et al. NOD2/CARD15 genotype and phenotype differences between Ashkenazi and Sephardic Jews with Crohn's disease. Am J Gastroenterol 2004; 99: 1134-1140.

30. Tukel T, Shalata A, Present $\mathbf{D}$ et al. Crohn's disease: frequency and nature of CARD15 mutations in Ashkenazi and Sephardic/Oriental Jewish families. Am J Hum Genet 2004; 74: 623-636.

31. Papp M, Altorjay I, Dotan $\mathbf{N}$ et al. New serological markers for inflammatory bowel disease are associated with earlier age at onset, complicated disease behavior, risk for surgery, and NOD2/CARD15 genotype in a Hungarian inflammatory bowel disease cohort. Am J Gastroenterol 2008; 103: 665-681.

32. Picornelli Y, Mai L, Taylor K et al. TNFSF 15 is an ethnic-specific inflammatory bowel disease gene. Inflamm Bowel Dis 2007; 13: 1333-1338.

33. Jass JR. Classification of colorectal cancer based on correlation of clinical, morphological and molecular features. Histopathology 2007; 50: 113-130.

34. Phipps AI, Limburg PJ, Baron JA et al. Association between molecular subtypes of colorectal cancer and patient survival. Gastroenterology 2015; 148: 77-87.

35. Fearon ER. Molecular genetics of colorectal cancer. Ann Rev Pathol 2011; 6: 479-507.

36. Balfour Sartor R. Genetic and environmental interactions shape the intestinal microbiome to promote inflammatory bowel disease versus mucosal homeostasis. Gastroenterology 2010; 139: 1816-1833.

37. Braun J. Targan SR. Multiparameter analysis of immunogenetic mechanisms in clinical diagnosis and management of inflammatory bowel disease. Adv Exp Med Biol 2006; 579: 209-218.

38. Fedorak RN. Probiotics in the management of inflammatory bowel diseases. Am J Gastroenterol 2007; 102: S22-S28.

39. Fiscella K, Winters $\mathbf{P}$, Tancredi $\mathbf{D}$ et al. Racial disparity in death from colorectal cancer: Does Vitamin D deficiency contribute? Cancer 2011; 117: 1061-1069.

40. Owyang C, Wu GD. The gut microbiome in health and disease. Gastroenterology 2014; 146: 1433-1436.

Received April 30, 2015. Accepted September 20, 2015. 